Irish Math. Soc. Bulletin

Number 77, Summer 2016, 51-60

ISSN 0791-5578

\title{
On Yamabe Soliton
}

\author{
SATYABROTA KUNDU
}

\begin{abstract}
The purpose of the paper is to prove that if the metric of a 3-dimensional $\alpha$-Sasakian structure on a complete Riemannian manifold is a Yamabe soliton then it is of constant curvature. We also derive some properties of the flow vector field $U$ of the Yamabe soliton together with an example of an $\alpha$-Sasakian manifold admitting Yamabe soliton.
\end{abstract}

\section{INTRODUCTION}

A well known question in differential geometry is whether a given compact connected Riemannian manifold is necessarily conformally equivalent to one having constant scalar curvature. This problem was formulated by Yamabe in 1960[13] and is known as the Yamabe problem. Yamabe gave a purported proof of the affirmative answer, but Trudinger in 1968[14] found an error, and then was able to correct the proof of Yamabe for the case when the scalar curvature is non-positive. Aubin improved Trudinger's result but the remaining cases were solved by Schoen using positive mass theorem.

Another motivation for considering the Yamabe problem is conformal geometry itself. Riemannian differential geometry attempted to generalize the highly successful theory of compact surfaces. From the earliest days, conformal changes of metric played an important role in surface theory. For instance, it is a consequence of the uniformization theorem of complex analysis that one can find a conformal change of metric which makes the scalar curvature constant. This led to the Yamabe problem.

Several years ago, the notion of Yamabe flow was introduced by Richard Hamilton (see [5], [6]) as a tool for constructing metrics of constant scalar curvature in a given conformal class of Riemannian

2010 Mathematics Subject Classification. 53C15, 53C21, 53C25.

Key words and phrases. $\alpha$-Sasakian 3-Metric, Yamabe Soliton, Infinitesimal Contact Transformation.

Received on 21-8-2015; revised 18-12-2015. 
metrics on $\left(M^{n}, g\right)(n \geq 3)$. It can also be explained as negative $L^{2}$ gradient flow of the (normalized) total scalar curvature, restricted to a given conformal class it can be interpreted as deforming a Riemannian metric to a conformal metric of constant scalar curvature, when this flow converges. Complete shrinking gradient Yamabe solitons under the assumptions of suitable scalar curvature (resp. Ricci tensor) have finite topological type. On a smooth Riemannian manifold, Yamabe flow can be defined as the evolution of the Riemannian metric $g_{0}$ in time $t$ to $g=g(t)$ by means of the equation

$$
\frac{\partial}{\partial t} g=-r g, \quad g(0)=g_{0},
$$

where $r$ denotes the scalar curvature corresponds to $g$. Yamabe soliton can be defined on a Riemannian manifold by a vector field $U$ (known as Flow Vector field) satisfying:

$$
£_{U} g=(c-r) g,
$$

where $£_{U}$ denotes the Lie-derivative operator along the direction of $U$ and the constant $c=-\dot{\sigma}\left(g_{0}\right)$ (see Chow et al. [3]). Similar to Ricci Soliton, Yamabe Soliton can be considered as a special solution of the Yamabe flow. In Mathematical Physics, Yamabe flow relates to the fast diffusion case of the plasma equation. Recently, Sharma (refer to [11]) have studied Yamabe soliton on 3-dimensional Sasakian metric on a complete manifold. Since $\alpha$-Sasakian is a generalization of Sasakian manifold, we are interested to study 3-dimensional $\alpha$ Sasakian manifold when its metric is a Yamabe soliton. We deduce some properties of the flow vector field $U$ of the Yamabe soliton.

\section{PRELIMINARIES}

An odd-dimensional differentiable manifold $\left(M^{n}, g\right)$ may admit an almost contact metric structure $(\Phi, \xi, \eta, g)$ consisting of a Reeb vector field $\xi$, a $(1,1)$-tensor field $\Phi$ and a Riemannian metric $g$ satisfying

$$
\begin{aligned}
\Phi^{2} & =-I+\eta \otimes \xi, \quad \eta(\xi)=1, \\
g(\Phi X, \Phi Y) & =g(X, Y)-\eta(X) \eta(Y), \forall X, Y \in \chi(M),
\end{aligned}
$$

where $\chi(M)$ represents the collection of all smooth vector fields on M.

Moreover, if the relation

$$
d \eta(X, Y)=g(\Phi X, Y),
$$


holds for arbitrary smooth vector fields $X$ and $Y$, then we call such a structure a contact metric structure and the manifold with that structure is said to be contact metric manifold. As a consequence of this, the following relations hold:

$$
\Phi \xi=0, \quad \eta \circ \Phi=0, \quad d \eta(\xi, X)=0, \quad g(\Phi X, Y)=-g(X, \Phi Y),
$$

$\forall X \in \chi(M)$. For details we refer to Blair [1].

An almost contact structure on $M$ is said to be an $\alpha$-Sasakian manifold, $\alpha$ being a non-zero constant, if

$$
\left(\nabla_{X} \Phi\right) Y=\alpha(g(X, Y) \xi-\eta(Y) X), \forall X, Y \in \chi(M)
$$

holds. As a consequence, it follows that:

$$
\begin{aligned}
\nabla_{X} \xi & =-\alpha \Phi X, \\
\left(\nabla_{X} \eta\right) Y & =-\alpha g(\Phi X, Y), \forall X, Y \in \chi(M) .
\end{aligned}
$$

If $\alpha=1$, then the $\alpha$-Sasakian structure reduces to Sasakian manifold, thus $\alpha$-Sasakian structure may be considered as a generalization of Sasakian one. In other words, Sasakian manifold is a particular case of $\alpha$-Sasakian manifold. Also in a 3-dimensional $\alpha$-Sasakian manifold the following relations are true:

$$
\begin{aligned}
R(X, Y) \xi & =\alpha^{2}\{\eta(Y) X-\eta(X) Y\}, \\
S(X, \xi) & =2 \alpha^{2} \eta(X), \\
Q \xi & =2 \alpha^{2} \xi, \quad \forall X, Y \in \chi(M),
\end{aligned}
$$

where $R$ is the Riemannian curvature tensor and $Q$ is the Ricci operator associated with the $(0,2)$ Ricci tensor $S$. For details we refer to [7].

Definition 2.1. (1])In an almost contact Riemannian manifold, if an infinitesimal transformation $U$ satisfies

$$
\left(£_{U} \eta\right)(X)=\sigma \eta(X), \quad \forall X \in \chi(M)
$$

for a scalar function $\sigma$, then we call it an infinitesimal contact transformation. If $\sigma$ vanishes identically, then it is called an infinitesimal strict transformation.

Definition 2.2. A vector field $U$ in an n-dimensional Riemannian manifold $(M, g)$ is said to be conformal if

$$
£_{U} g=2 \nu g,
$$


for some smooth function $\nu$ on $M$. Moreover, a conformal vector field satisfies

$$
\begin{gathered}
\left(£_{U} S\right)(X, Y)=-(n-2) g\left(\nabla_{X} D \nu, Y\right)+(\Delta \nu) g(X, Y) \\
£_{U} r=-2 \nu r+2(n-1) \Delta \nu
\end{gathered}
$$

where $D$ is the gradient operator and $\Delta=-$ div.D is the Laplacian operator of $\mathrm{g}$. For details, we refer to Yano [12].

Before proceeding for the main results let us state the following lemmas:

Lemma 2.1. In an $\alpha$-Sasakian manifold, the following relations are valid:

$$
\begin{aligned}
& \text { (i): } \eta\left(£_{U} \xi\right)=\frac{r-c}{2}, \\
& \text { (ii): }\left(£_{U} \eta\right)(\xi)=\frac{c-r}{2} \text {. } \\
& \text { (iii): } \nu=\frac{c-r}{2} .
\end{aligned}
$$

Proof. The proof (i) and (ii) readily follows from the definition of the Yamabe soliton on a Riemannian manifold. Since $U$ is a conformal vector field, therefore using (2.11) and (i), we have the desired result (iii).

Lemma 2.2. In an $\alpha$-Sasakian 3-Metric, the Ricci tensor $S$ is given by

$$
S=\left(\frac{r}{2}-\alpha^{2}\right) g+\left(3 \alpha^{2}-\frac{r}{2}\right) \eta \otimes \eta .
$$

Proof. We recall that the Riemannian curvature tensor in a 3-dimensional Riemannian manifold is given by

$$
\begin{aligned}
R(X, Y) Z= & g(Y, Z) Q X-g(X, Z) Q Y+S(Y, Z) X-S(X, Z) Y \\
& -\frac{r}{2}\{g(Y, Z) X-g(X, Z) Y\}
\end{aligned}
$$

where $r$ is the Ricci scalar curvature and $X, Y, Z \in \chi(M)$. Replacing $Z$ with $\xi$ in (3.1) and recalling (2.8), yields

$$
\eta(Y) Q X-\eta(X) Q Y+\left(\alpha^{2}-\frac{r}{2}\right)\{\eta(Y) X-\eta(X) Y\}=0 .
$$

Again, replacing $Y$ with $\xi$ and thereby using (2.9), we get the desired result. 


\section{MAIN RESULTS}

Putting the value of $\nu$ in $(2.12)$ and (2.13), one obtains

$$
\left(£_{U} S\right)(X, Y)=\frac{1}{2}\left[g\left(\nabla_{X} D r, Y\right)-(\Delta r) g(X, Y)\right],
$$

and

$$
£_{U} r=-2 \Delta r-r(c-r) .
$$

Since $g$ is an Yamabe Soliton, taking the Lie-derivative of $(2.14)$ in the direction of $U$ and using (3.1) and (3.2), yields

$$
\begin{aligned}
g\left(\nabla_{X} D r, Y\right)= & -\left[\Delta r+2 \alpha^{2}(c-r)\right] g(X, Y) \\
+ & {[2 \Delta r+r(c-r)] \eta(X) \eta(Y) } \\
& +\left(6 \alpha^{2}-r\right)\left[\left(£_{U} \eta\right)(X) \eta(Y)+\left(£_{U} \eta\right)(Y) \eta(X)\right] .
\end{aligned}
$$

Since $\xi$ is killing, therefore $\xi r=0$. Differentiating covariantly along the direction of an arbitrary vector field $X$, one obtains $g\left(\nabla_{X} D r, \xi\right)=$ $(\alpha \Phi X) r$. Replacing $Y$ with $\xi$ in (3.3) and using the foregoing equation together with the lemma provides

$$
\alpha(\Phi X) r=\left\{\Delta r+(c-r)\left(\alpha^{2}+\frac{r}{2}\right)\right\} \eta(X)+\left(6 \alpha^{2}-r\right)\left(£_{U} \eta\right) X .
$$

Putting $X=\xi$, in above and using the lemma(ii) we obtain

$$
\Delta r=-4 \alpha^{2}(c-r)
$$

From (3.4) and (3.5), we can deduce

$$
\left(6 \alpha^{2}-r\right)\left(£_{U} \eta\right) X=\alpha(\Phi X) r+\frac{1}{2}(r-c)\left(r-6 \alpha^{2}\right) \eta(X) .
$$

Feeding (3.5) and $(3.6)$ in $(3.3)$, yields

$$
\nabla_{X} D r=2 \alpha^{2}(c-r)(X-\eta(X) \xi)-\alpha((\phi X) r) \xi-\alpha \eta(X)(\Phi D r) \text {. }
$$


Differentiating covariantly along the direction of $Y$, we obtain

$$
\begin{aligned}
\nabla_{Y} & \nabla_{X} D r= \\
& -2 \alpha^{2}(Y r)(X-\eta(X) \xi) \\
& +2 \alpha^{2}(c-r)\left\{\nabla_{Y} X+\alpha g(\Phi Y, X) \xi-\eta\left(\nabla_{Y} X\right) \xi+\alpha \eta(X)(\Phi Y)\right\} \\
& +\alpha\left(\nabla_{Y} \nabla_{\Phi X} r\right) \xi \\
& -\alpha^{2}((\Phi X) r) \Phi Y \\
& -\alpha \eta(X)\left\{\alpha g(Y, D r) \xi-\alpha \eta(X) \Phi D r+\Phi\left(\nabla_{Y} D r\right)\right\} \\
& -\alpha\left\{-\alpha g(\Phi Y, X)+\eta\left(\nabla_{Y} X\right)\right\}(\Phi D r) .
\end{aligned}
$$

Replacing $X$ and $Y$ in above, one obtains

$$
\begin{aligned}
\nabla_{X} \nabla_{Y} D r= & -2 \alpha^{2}(X r)(Y-\eta(Y) \xi) \\
+ & 2 \alpha^{2}(c-r)\left\{\nabla_{X} Y+\alpha g(\Phi X, Y) \xi\right. \\
& \left.-\eta\left(\nabla_{X} Y\right) \xi+\alpha \eta(Y)(\Phi X)\right\} \\
+ & \alpha\left(\nabla_{X} \nabla_{\Phi Y} r\right) \xi-\alpha^{2}((\Phi Y) r) \Phi X \\
- & \alpha \eta(Y)\left\{\alpha g(X, D r) \xi-\alpha \eta(Y) \Phi D r+\Phi\left(\nabla_{X} D r\right)\right\} \\
- & \alpha\left\{-\alpha g(\Phi X, Y)+\eta\left(\nabla_{X} Y\right)\right\}(\Phi D r) .
\end{aligned}
$$

Again from (3.7), we have

$$
\begin{aligned}
\nabla_{[Y, X]} D r= & 2 \alpha^{2}(c-r)\left\{\nabla_{Y} X-\nabla_{X} Y-\eta\left(\nabla_{Y} X\right) \xi+\eta\left(\nabla_{X} Y\right) \xi\right\} \\
& +\alpha\left\{\left(\Phi \nabla_{Y} X\right) r-\left(\Phi \nabla_{X} Y\right) r\right\} \xi \\
- & \alpha\left\{\eta\left(\nabla_{Y} X\right)-\eta\left(\nabla_{X} Y\right)\right\}(\Phi D r) .
\end{aligned}
$$

Also, the Riemannian curvature tensor $R$ is given by,

$$
R(X, Y) D r=\left[\nabla_{X}, \nabla_{Y}\right] D r-\nabla_{[X, Y]} D r .
$$

Feeding the equations (3.8), (3.9) and (3.10) in the foregoing formula, then we obtain on contracting the above over $X$ and recalling the skew-symmetric property of $\Phi$ together with $\xi r=0$ and (3.7),

$$
S(X, D r)=-\alpha g\left(\Phi \nabla_{e_{i}} D r, e_{i}\right) \eta(X) .
$$

Combining the above with Lemma(2.1) and recalling Lemma(2.3), one obtains

$$
\left(r-2 \alpha^{2}\right) X r=0 .
$$

which shows the scalar curvature $r$ is constant. Hence appealing to (3.5), yields $r=c$. Thus we can state: 
Theorem 3.1. If the metric of a 3-dimensional $\alpha$-Sasakian manifold is a Yamabe soliton, then it is of constant scalar curvature $c$.

Hence (1.1) reduces to $£_{U} g=0$, i.e. $U$ is killing. Differentiating covariantly along an arbitrary vector field $X$, we have $\nabla_{X} £_{U} g=0$. The identity

$$
\left(\nabla_{X} £_{U} g\right)(Y, Z)=g\left(\left(£_{U} \nabla\right)(X, Y), Z\right)+g\left(\left(£_{U} \nabla\right)(X, Z), Y\right),
$$

can be deduced from the formula [for details we refer to [12]],

$$
\begin{gathered}
\left(£_{U} \nabla_{X} g-\nabla_{X} £_{U} g-\nabla_{[U, X]} g\right)(Y, Z) \\
=-g\left(\left(£_{U} \nabla\right)(X, Y), Z\right)-g\left(\left(£_{U} \nabla\right)(X, Z), Y\right),
\end{gathered}
$$

which implies,

$$
g\left(\left(£_{U} \nabla\right)(Z, X), Y\right)+g\left(\left(£_{U} \nabla\right)(Z, Y), X\right)=0 .
$$

By the combinatorial combination of the above together with the skew-symmetric property of $\phi$, yields

$$
\left(£_{U} \nabla\right)(Y, Z)=0 \text {. }
$$

Taking $Y=Z=\xi$, we obtain $\left(£_{U} \nabla\right)(\xi, \xi)=0$. Hence, with the geodesic property of $\xi$, the following identity

$$
\left(£_{U} \nabla\right)(X, Y)=\nabla_{X} \nabla_{Y} U-\nabla_{\nabla_{X} Y} U+R(U, X) Y,
$$

yields $R(U, \xi) \xi+\nabla_{\xi} \nabla_{\xi} U=0$, which concludes that $U$ is Jacobi along the direction of $\xi$. Since $r$ is constant $(=\mathrm{c})$, from (3.4), we can say that either $r=6 \alpha^{2}$ or $r \neq 6 \alpha^{2}$. In the former case, from Lemma(2.2) we can conclude that $S=2 \alpha^{2} g$ i.e. $M$ is an Einstein manifold and being of dimension 3 it is of constant curvature $\alpha^{2}$. Thus we can state as follows:

Theorem 3.2. If the metric of a 3-dimensional $\alpha$-Sasakian manifold is a Yamabe soliton, then the flow vector field $U$ is killing and is Jacobi along the direction of $\xi$. In particular if $c=6 \alpha^{2}$, the manifold reduces to an Einstein manifold.

If $c(=r) \neq 6 \alpha^{2}$, then recalling equation (3.6) we conclude $£_{U} \eta=$ 0 . Thus from the definition of an infinitesimal contact transformation, the scalar function $\sigma$ vanishes identically and hence we can state:

Theorem 3.3. If the metric of a 3-dimensional $\alpha$-Sasakian manifold is a Yamabe soliton, then the infinitesimal contact transformation of the conformal vector field is strict. 
A brief computation on using the result of for a 3-dimensional $\alpha$ Sasakian manifold that the $\phi$-sectional curvature equals $\frac{1}{2}\left(r-4 \alpha^{2}\right)$. By virtue of theorem (3.1), we get the following:

Theorem 3.4. For a 3-dimensional $\alpha$-Sasakian manifold the $\phi$ sectional curvature(sectional curvature with respect to a plane orthogonal to $\xi$ ) is constant and equals to $-\alpha^{2}$.

\section{EXAMPLE OF AN $\alpha$-SASAKIAN 3-METRIC AS YAMABE SOLITON}

Let us consider the 3-dimensional Riemannian manifold $M=\mathbb{R}^{3}$ with a rectangular cartesian coordinate system $\left(x_{i}\right)$.

Let us choose the vector fields $\left\{E_{1}, E_{2}, E_{3}\right\}$ as

$$
E_{1}=\frac{\partial}{\partial x_{1}}, \quad E_{2}=-2 \alpha \frac{\partial}{\partial x_{2}}, \quad E_{3}=x_{2} \frac{\partial}{\partial x_{1}}+x_{3} \frac{\partial}{\partial x_{3}},
$$

$\alpha$ a non-zero constant. Thus, $\left\{E_{1}, E_{2}, E_{3}\right\}$ forms a basis of $\chi(M)=$ $\chi\left(\mathbb{R}^{3}\right)$.

Let $g$ be the Riemannian metric on $\chi\left(\mathbb{R}^{3}\right)$ defined by

$$
\left\{\begin{array}{l}
g\left(E_{1}, E_{1}\right)=g\left(E_{2}, E_{2}\right)=g\left(E_{3}, E_{3}\right)=1 \\
g\left(E_{1}, E_{2}\right)=g\left(E_{1}, E_{3}\right)=g\left(E_{2}, E_{3}\right)=0 .
\end{array}\right.
$$

Let $\xi=E_{1}$ be the vector field associated with the 1-form $\eta$. The $(1,1)$-tensor field $\phi$ be defined by,

$$
\phi\left(E_{1}\right)=0, \quad \phi\left(E_{2}\right)=-E_{3}, \quad \phi\left(E_{3}\right)=E_{2} .
$$

Since, $\left\{E_{1}, E_{2}, E_{3}\right\}$ is a basis, any vector fields $X$ and $Y$ in $M$ can be uniquely expressed as

$$
X=X^{1} E_{1}+X^{2} E_{2}+X^{3} E_{3} \text { and } Y=Y^{1} E_{1}+Y^{2} E_{2}+Y^{3} E_{3},
$$

where $X^{i}, Y^{i}(i=1,2,3)$ are smooth functions over $M$.

Now using the linearity of $\phi$ and $g$, and taking $\xi=E_{1}$ we have,

$\eta(\xi)=1, \quad \phi^{2} X=-X+\eta(X) \xi, \quad g(\phi X, \phi Y)=g(X, Y)-\eta(X) \eta(Y)$, for any vector fields $X$ and $Y$ in $M$. Thus $(\phi, \xi, \eta, g)$ defines an almost contact metric structure on $M$.

Let $\nabla$ be the Levi-Civita connection with respect to the Riemannian metric $g$. Then we have,

$$
\left[E_{2}, E_{3}\right]=-2 \alpha e_{1}, \quad\left[E_{1}, E_{2}\right]=0, \quad\left[E_{1}, E_{3}\right]=0 .
$$


By using Koszul's formulae (see [9]), we have

$$
\begin{array}{lll}
\nabla_{E_{1}} E_{3}=-\alpha E_{2}, & \nabla_{E_{1}} E_{2}=\alpha E_{3}, & \nabla_{E_{1}} E_{1}=0, \\
\nabla_{E_{2}} E_{3}=-\alpha E_{1}, & \nabla_{E_{2}} E_{1}=\alpha E_{3}, & \nabla_{E_{2}} E_{2}=0, \\
\nabla_{E_{3}} E_{1}=-\alpha E_{2}, & \nabla_{E_{3}} E_{2}=\alpha E_{1}, & \nabla_{E_{3}} E_{3}=0 .
\end{array}
$$

Also, the Riemannian curvature tensor $R$ is given by

$$
R(X, Y) Z=\nabla_{X} \nabla_{Y} Z-\nabla_{Y} \nabla_{X} Z-\nabla_{[X, Y]} Z \text {. }
$$

Then,

$$
\begin{array}{ll}
R\left(E_{1}, E_{2}\right) E_{2}=\alpha^{2} E_{1}, & R\left(E_{1}, E_{3}\right) E_{3}=\alpha^{2} E_{1}, \\
R\left(E_{2}, E_{1}\right) E_{1}=\alpha^{2} E_{2}, & R\left(E_{2}, E_{3}\right) E_{3}=-3 \alpha^{2} E_{2}, \\
R\left(E_{3}, E_{1}\right) E_{1}=\alpha^{2} E_{3}, & R\left(E_{3}, E_{2}\right) E_{2}=-3 \alpha^{2} E_{3},
\end{array}
$$

and

$$
R\left(E_{1}, E_{2}\right) E_{3}=R\left(E_{2}, E_{3}\right) E_{1}=R\left(E_{3}, E_{1}\right) E_{2}=0 .
$$

Then, the Ricci tensor $S$ is given by

$$
\begin{array}{lll}
S\left(E_{1}, E_{1}\right)=2 \alpha^{2}, & S\left(E_{2}, E_{2}\right)=-2 \alpha^{2}, & S\left(E_{3}, E_{3}\right)=-2 \alpha^{2}, \\
S\left(E_{1}, E_{2}\right)=0, & S\left(E_{1}, E_{3}\right)=0, & S\left(E_{2}, E_{3}\right)=0 .
\end{array}
$$

It is easy to verify that the above structure satisfies the conditions of $\alpha$-Sasakian manifold. Also, the constructed metric of the $\alpha-$ Sasakian manifold is Yamabe soliton. It is seen that the scalar curvature $r=-2 \alpha^{2}$, implies that the infinitesimal contact transformation of the flow vector field is strict.

\section{REFERENCES}

[1] Blair, D.E., Riemannian Geometry of Contact and Symplectic Manifolds, Birkhäuser, Boston, 2010.

[2] Chow, B., Knopf, D., The Ricci flow: An introduction, Mathematical Surveys and Monographs, AMS, Providence, RI, 2004.

[3] Chow, B., Lu, P. and Ni, L., Hamilton's Ricci flow, Graduate Sudies in Math., Vol. 77, American Math. Soc. Science Press, 2006.

[4] Guilfoyle, B. S., Einstein metrics adapted to a contact structure on 3manifolds, Preprint, http://arXiv.org/abs/math/0012027, 2000.

[5] Hamilton, R.S., Lectures on geometric flows, unpublished manuscript, 1989.

[6] Hamilton, R.S., The Ricci flow on surfaces, Mathematics and general relativity (Santa Cruz, CA, 1986), 237-262, Contemp. Math. 71, American Math. Soc., 1988.

[7] Janssens, D. and Vanhecke, L., Almost Contact Structures and Curvature Tensors, Kodai Math. J., 4 (1981), 1-27.

[8] Perelman, G., The entropy formula for the Ricci flow and its geometric applications, Preprint, <http://arXiv.org/abs/math.DG/02111159>. 
[9] Schouten, J. A., Ricci Calculus, Springer-Verlag, Berlin, 2nd Ed.(1954), pp. 332.

[10] Sharma, R., Certain results on K-contact and $(\kappa, \mu)$-contact manifolds, $J$. Geom., 89(2008) 138-147.

[11] Sharma, R., A 3-Dimensional Sasakian Metric as a Yamabe Soliton, International Journal of Geometric Methods in Modern Physics, Volume 09, Issue 04, June 2012 .

[12] Yano, K., Integral Formulas in Riemannian Geometry, Marcel Dekker, New York, 1970.

[13] Yamabe, H.,On a deformation of Riemannian structures on compact manifolds, Osaka Math. J., 12:21-37, 1960.

[14] Trudinger, S. N.,Remarks on the deformation of Riemannian structures on compact manifolds, Ann. Scuolo Norm. Sup. Pisa(3), 22:265-274, 1968.

Satyabrota Kundu has an MSc and PhD from the Univeristy of Calcutta, and now works as Assistant Professor in the Department of Mathematics at J. K. College, Purulia, West Bengal, India. His research is mainly focussed on different structures on Riemannian Manifolds. At present he is working on Soliton theory (Ricci and Yamabe solitons).

Department of Mathematics, J. K. College, Purulia-723101, West BENGAL, INDIA

E-mail address: satyacaluniv@gmail.com 